Meeting of the Aristotelian Society at 21, Gower Street, W.C. 1, on June 12th, 1922, at 8 p.m.

\title{
XI.-GEOMETRY AND REALITY.
}

\section{By Thomas Greenwood.}

For many centuries, geometry and mechanics had the most brilliant fortune as rational sciences. But the discovery of nonEuclidian geometry, and the momentous revolution brought about in the field of natural philosophy during these last years, have thrown strong doubts on the self-evidence of the fundamental concepts of geometry, and the basic notions of Newtonian mechanics. It is argued now, that only æsthetical considerations and psychological reasons of formal economy and utility could justify the privileged position of the axioms of these sciences. The practice, for example, of seeing in a distance two marked positions on a practically rigid body is something which is lodged deeply in our habit of thought. We are accustomed further to regard three points as being situated on a straight line, if their apparent positions can be made to coincide for observation with one eye, under suitable choice of our place of observation. If, in pursuance of our habit of thought, we now supplement the propositions of Euclidian geometry by the single proposition that two points on a practically rigid body always correspond to the same distance or line-interval, independently of any changes in position to which we may subject the body, the propositions of Euclidian geometry then resolve themselves into propositions on the possible relative position of practically rigid bodies.*

One is led then to suppose that the truths of geometry and mechanics reach a unique level. As Professor Painlevé says, there is no essential difference between geometry and mechanics ; $†$

* Einstein, Relativity, Chap. I.

† Painler6, La Mécanique. in Méthode des Sciences, vol. i. 
both are experimental sciences, although their developments have been different; the axioms of pure geometry are nothing else but the refined form of the properties of natural bodies. And Böcher writes: "Geometry becomes the simplest of the natural sciences, and its axioms are of the nature of physical laws, to be tested by experience and to be regarded as true only within the limits of the errors of observation."*

Professor Einstein has a different opinion, when he writes in his book on Relativity that the concept "true" does not tally with the assertions of pure geometry, because by the word "true" we are eventually in the habit of designating always the correspondence with a "real" object; geometry, however, is not concerned with the relations of the ideas involved in it to objects of experience, but only with the logical connexion of the ideas among themselves.t Poincare had already said: "Geometry is not an experimental science; experience forms merely the occasion for our reflecting upon the geometrical ideas which pre-exist within us." $\ddagger$

And Professor Eddington answering the question whether it is true to say that " any two sides of a triangle are together greater than the third side," says he is quite unable to say whether this proposition is true or not. "I can deduce it," he continues, "from certain other propositions still more elementary, the axioms; if these are true, the proposition is true; if the axioms are not true, the proposition is not true universally; whether the axioms are true or not, I cannot say, and it is outside $\mathrm{my}$ province to consider. But for reasons which I do not profess to understand, my friend the physicist is more interested in Euclidian geometry and is continually setting us problems in it." $\$$

All these conflicting opinions are unilateral. For the

* Bulletin Amer. Math. Soc. [2], 2 (1904), p. 124.

+ Einstein, Relativity, Chap. I.

‡ "Foundations of Geometry," in Monist, 9 (1899), p. 41.

$\$$ Eddington, Space, Time and Gravitation, Chap. I. 
concention of physicists that no system of geometry can be taken as true is based on the fact that they consider geometry with reference to natural phenomena, and fail to take it as an independent science, formally irrelevant to a description of the universe. We must remember here, that truth has two aspects : a formal and a material aspect. A proposition is said to be formally true, either when its terms are not contradictory, or when it can be deduced, by means of reasoning, from a coherent system of primitive notions and axioms relatively to which it is said to be true. The material aspect of truth is of a more complex character; for we have to deal here not only with thought; but also with facts, that is to say with data of intuition and experience. Material truth requires the agreement of thinking with experience, while formal truth is caused by the self-agreement of thought. It follows that a proposition can be formally true and materially false; for instance, when we say that " the hypervolume of a hypersphere is equal to its volume multiplied by one-fourth of its radius" this statement is formally true, but it is not verifiable by experience and is therefore materially false. This distinction between the two aspects of truth is of great importance, because it gives the clue to the specific difference between pure geometry and applied mechanics. It will be observed also that we take truth in the scholastic sense of adequatio rei et intellectus; but we extend the meaning of the word "res" not only to objerts of the external world, but also to objects created by thought.

Now, although the ultimate basis of geometry is empirical, it must be acknowledged that the active role of experience ceases when the primitive notions and postulates of geometry are established. Geometry belongs then to the conceptual order and constitutes what is called "axiomatic geometry," a kind of hypothetico-deductive system. As such, pure geometry sets out from certain primitive notions, such as " point," "straight line," "plane," which are not defined and with which we are able to associate more or less definite ideas, and from certain 
simple propositions or axioms, or postulates, which, in virtue of these ideas, we are inclined to accept as respecting the laws of thought. These axioms state certain relations between the primitive notions, which are thus supplemented and universally characterized. When, on the basis of a logical process common to all sciences, the justification of which we feel ourselves compelled to admit, all remaining propositions are shown to follow from those axioms, they are proved.

But if there is no difficulty about the truth of a geometrical proposition deduced from a coherent set of axioms, what about the truth of the axioms themselves? This question is unanswerable by the methods of geometry; but it is not in itself entirely without meaning, as Einstein eays. Without going far into the essence of geometrical axioms, we can say that they are true in so far as they express possible relations between primitive notions, undefined in the realm of geometry, but having a limited field of indetermination by reason of the intuitive data which preside at their formation. Geometrical axioms are true, because in the conceptual order, thought binds itself with the results of its normal activity.

The case of physical axioms is different, for they are always subject to some discussion and susceptible of being overthrown or contradicted by some new discoveries, while geometrical axioms are immutable in themselves. The reason of the revisibility of physical postulates lies in the very object of physical science. Having observed a series of natural phenomena, the physicist has to classify them in order to stabilize bis observations and deduce from them the laws which are to constitute science. But the external world does not give our senses any presentations of natural laws. Like the ancient Sphynx, nature does not give away her secrets: the mind has to guess them. How is it done? How do we proceed successfully from particular observations to those universal laws presenting the harmony of the universe? The only way is to form hypotheses based on analogies: by an act of imagination, human mind can 
suppose between the observed facts some possible relations which are tried afterwards by means of experimentation. Thus Kepler, led by metaphysical considerations, thought that the stars must obey certain laws in their motion; and only much later was he able to verify that his observations coincided with the constructions of his imagination.

There is no difficulty about the truth of what we observe and what we experience because of the simplicity of senseperception. The whole question of truth, in physics, lies then entirely in the modality of the hypotheses. Although a physical hypothesis is an act of imagination, and therefore subject to the laws of thought, it is by no means a free creation of the mind, a convention similar to geometrical axioms. For if the latter have only to be coherent between themselves in a definite system, and are irrelevant to their actual interpretation, the former must satisfy a further condition, which is far more complicated: a physical hypothesis must necessarily lead to the explanation of the group of phenomena through which it developed, and also of the new phenomena which observation will bring in that group. Nature cannot obey the fancies of our imagination; we have to find out her own laws and obey them first. If geometry deals with formal relations between indefinable concepts without considering the content of these concepts, the physicist has especially to care about that content itself. $\mathrm{He}$ is not at liberty to play with mathematical symbols; for he has to struggle with a reality which sets limits, wide enough however, to his imagination.

For instance, the compression of gases is at first proportional to the exerted pressure; but then, parting with its mathematical expression, it becomes smaller and smaller. In the same way, it is always possible to increase steadily the speed of a train; but the stability of the railroad, the strength of the materials in use, the structural elements of the engine, impose an irrefragable limit upon the practicability of the calculations. Then again, mathematics can build up a most wonderful 
theory of hyperpolyhedra, but there is no matter to which the conclusions of such systems can be applied. It is a truism that the science of the possible is infinite; but it is the sanction of reality which gives a material value to an hypothesis.

Let us illustrate with a general example the contrast between geometry and physics. By means of the Euclidian geometry and the Galilean system of co-ordinates, Newton, considering space and time as two absolutes, had been able to give a mathematical expression to his immortal law of gravitation. Now the evolution of science has led Professor Einstein to give a different law of the universal attraction, by means of Minkowski's four-dimensional Universe and Riemann's generalization of the Gaussian system of co-ordinates. The difference of the two laws may be expressed analytically, in polar co-ordinates for a particle of gravitational mass, $m$, as follows: Any particle of light pulse moves so that the integral of the interval, $d s$, between two points of its path, in four dimensions, is stationary where

$$
\begin{aligned}
& d s^{2}=-\gamma^{-1} d r^{2}-r^{2} d \theta^{2}+\gamma d t^{2} \text { (Einstein), } \\
& d s^{2}=-d r^{2}-r^{2} d \theta^{2}+\gamma d t^{2} \text { (Newton), }
\end{aligned}
$$

considering that $\gamma=1-2 m / r$.

The two laws differ then by the factor multiplying the term $d r^{2}$. Which corresponds to the external world? Three crucial tests were predicted by Einstein to meet this question : (1) the determination of the discrepancy of the perihelion of Mercury, (2) the deflection of a ray of light passing near a great mass like the sun, (3) the displacement of the lines of the spectrum towards the red in a gravitational field. The story of these historical tests is well known; with certain restrictions for the third one, they all verify Einstein's law, which is then to be considered as the true law of gravitation, while Newton's law is but a limiting case of Einstein's General Principle of Relativity. 
But if these laws are considered as logical consequences of two hypothetico-deductive systems starting with different postulates, both of them are formally true; and their difference is but a consequence of the fundamental conceptions underlying Newton's and Einstein's mechanics, which, however, in themselves are also true. It is the content of experience, expressed by these laws, which influences their material truth.

The contrast between the methods of geometry and physics based on the distinction between formal and material truth, shows easily how meaningless is the question of the true geometry of the universe.

From Lobatchefski to Einstein, a long series of mathematicians thought it possible to determine the geometry of the universe. For them the question: According to the laws of what geometry do the natural bodies behave? has a precise meaning which could be worked out by experimental tests. In other words, the question whether Euclidian, or Lobatchefskian, or Riemannian geometry corresponds to the external world, can be answered experimentally, within the limits of errors of observation. We must only assume that light propagates in a straight line, and that every measure of length, whether geodesic or astronomical, is to be calculated by means of the propagation of light.

The experiment imagined by Lobatchefski and Bolyai, the originators of what Schweikart calls "astral geometry," was very simple. By measuring the three interior angles of a large triangle, they thought to verify whether that sum is equal, less or greater than two right angles. In the first case, space would be Euclidian; in the second case, it would be Lobatchefskian ; and in the third case, it would be Riemannian. And, as Legendre proved it, the verification for one triangle must stand good for any other triangle. Gauss had already attempted a similar experiment: while Kant was endeavouring to show the psychological character of spatial intuition, and deduce its physical meaning, Gauss had undertaken more 
accurate geodesic measurements of triangulation in the Hartz Valley.

But all these observations proved negative : space presented itself as Euclidian. Nevertheless there was an idea amongst men of science, that more accurate observations and the development of the mechanical consequences of non-Euclidian geometry with regard to astronomical problems, would certainly favour the legitimacy of non-Euclidian postulates as physical hypotheses. This was done by Einstein, when he built up his theory of Relativity by means of Riemann's geometrical conceptions. As a matter of fact, without the wonderful development of non-Euclidian geometry, Einstein's acbievement would have been impossible. But are we to conclude, with him, that the geometry of the universe is not Euclidian? This affirmation is simply too bold and premature, for it has no real import. And we must confess that Einstein's cosmological considerations on this topic are the least convincing portion of his work.

Euclidian geometry, says Einstein, must be abandoned, because in a system of reference rotating relatively to a system at rest, the geometrical behaviour of the bodies, which are affected hy the Fitzgerald-Lorentz contraction, does not correspond to Euclidian geometry. In simpler words, the path of moving bodies in nature is never a Euclidian straight line, because of the action of gravitational fields, which is always effective; the universe therefore, is not Euclidian.

The fallacy of this argument is easily seen, if we point out that because the path of a moving body is influenced by the presence of gravitational fields, it does not follow at all that the Euclidian straight line path is not there. As a matter of fact, it is there, although not actually followed by the moving body. Let us consider for a moment a photograph of the solar eclipse of May, 1919. We see plainly the maguificent curve described by the rays of light of remote stars passing near the sun; but at the same time, we see that there is a Euclidian 
straight line path between these very stars and the point where their rays of light touch the earth. It is not followed by these rays of light, because, according to the Principle of Least Action, the shortest way between two points in a spatiotemporal continuum is influenced by the presence of gravitational fields. And this is so far true that if the sun, which causes the gravitational field in this case, were taken further and further away, the path of these same rays of light would have a decreasing curvature, thus tending asymptotically towards a Euclidian straight line. That path would be adequately Euclidian, if per impossibile the sun and all other gravitational fields were removed from the universe.

Another argument familiar to relativists is that the geometry of the universe cannot be Euclidian, because Einstein's theory is based on Riemannian geometry, where space is curved and the straight line illimited but finite. This argument, however, looked through its adequate proportion, means only that Riemann's geometry is more convenient than any other for the description of the universe. In fact, there is a principle in the theory of groups of transformations, called the Principle of Equivalence, which enabled Poincaré, Klein and others to transpose any system of metrical geometry into any other. By means of a biunivocal correspondence, illustrated with an appropriate vocabulary, between two geometries, any Riemannian concept, for instance, is shown to be equivalent to a Euclidian concept. It follows then, that the group of natural phenomena explained by the theory of Relativity can be interpreted by means of Euclidian geometry: as axiomatic geometry alone makes no affirmations on the reality accessible to experience, but only axiomatic geometry completed with physical propositions, it is possible, whatever be the nature of reality, to keep Euclidian geometry. Geometry [G] does not enunciate anything on the behaviour of real objects, but geometry together with the sum [P] of physical laws; it is the sum $[\mathrm{G}+\mathrm{P}]$ which can be checked by experience. It is then 
always possible to take [G] as Euclidian and make appropriate assumptions with reference to some parts of $[\mathrm{P}]$; it is only necessary to take the remainder of $[\mathrm{P}]$ such as the sum $[\mathrm{G}+\mathrm{P}]$ is in agreement with experience. In the case of Relativity, however, it is more convenient and less complicated to describe the universe as Riemannian. Convenience is then the condition of the choice of the world's geometry; and we must draw the attention to the word "description" in physical science, which has the profoundest significance for Epistemology." Because a description is always by means of the accidents: the essence of the thing described is left untouched by this operation.

Now we come to the ontological aspect of our-argument. With all the essential difference between the object and method of geometry and of physics, there must be a close connexion between these two disciplines. Because, on the one hand, physical sciences cannot reach their actual degree of certitude without the help of mathematics, and on the other hand, mathematics would be useless if it had no practical value, considering also that its origin is enupirical. In fact, when the thinking person stops to reflect upon the fact that the existence of Neptune was pointed out to the astronomer before his telescope had noticed this wanderer in the remote heavens; when he learns that the mathematician by a theory related to the solution of the problem of finding the roots of an algebraic equation, is able to say that there are not more than thirty-two distinct types of crystals; when he remembers that the existence of wireless telegraphy is due to deductions of Maxwell by means of theorems that depend upon imaginary quantities; when, to give a last instance, he considers that the abstruse non-Euclidian geometry of Riemann and the tortuous theory of absolute

* D. Wrinch, "On certain Methodological Aspects of the Theory of Relativity," in Mind, April, 1922 ; and B. Russell, Introduction to Mathematical Philosophy. 
differential calculus of Ricci and Levi-Civita enabled Einstein to work out his momentous law of gravitation, which is, as says Sir J.J. Thomson, one of the highest achievements of human thought; he will undoubtedly endeavour to penetrate this mysterious riddle which has perplexed all the great seekers of the unknown: How is it possible that geometry and mathematics in general, which are constructions of the human mind, independent, in their structure and development, of all experience, adapt thernselves so wonderfully to the objects of reality? Is Reason able to discover by its sole activity, the very properties of the existing universe?

By denying the reality of matter for the benefit of extension, Descartes was led to the conclusion that geometry and mathematics in general (because of his invention of analytical geometry) are the science of Reality, the science which could penetrate the ultimate essence of its olject; and Nature would be completely known when the edifice of mathematics would be completed. And the modern style logicist, with all the restrictions he makes in the Cartesian doctrine, still holds that Reason is the ruler of things as well as the ruler of thought. For him, mathematics, mechanics, physics and every science which uses mathematical expressions are a developed aspect of logic; so that there is no incompatibility between the laws of chemistry, for instance, and the laws of thought, as Leibniz said, Dum Dents calculat fit mundus.

But on the other hand, we nust remember that when the mathematician tries his creative power of imagination on ideal constructions, he does not think of any practical utilization of the results he obtains. While to the physicist mathematical systems are operators enabling him to act more successfully on matter, to the mathematician the construction of an abstract theory is an end in itself independently of its applications. As Professor Bouasse says, the creator of new mathematical forms does not care whether his inventions correspond to some reality. The forms in themselves interest 
him more than anything else, for they enlarge the readyreckoner of mathematical forms. It is another question whether in a near or remote future, physical phenomena will consent to lodge in those structures. The algebraist prepares in advance sets of moulds which will be utilized by the physicist according to his convenience. But he does not think of that convenience when he makes them; although history shows us that many times the solution of a physical problem has led physicists to invent new unathematical forms, as for example, when the ideas of Faraday led Maxwell to the wathematical exposition of the electromagnetic theory.

The independence of mathematicians towards reality is shown by the fact that a great number of mathematical and specially of geometrical constructions do not tind or rather cannot have a corresponding reality. We have mentioned the theory of hyperpolyhedra which has no application in nature. In the same way it is impossible to give an adequate geometrical description of a flower. Nature is far more complicated than geometry and mathematics; so that if we are to describe the external world, mathematics must be supplemented with qualitative principles. It is certain then that mathematics are not at all co-extensive with reality: On the one hand, reality outruns them by its imprevisibility; and on the other hand, it is overstepped by them by all the distance between existence and possibility.

However, it is always possible to reconcile the real with that overwhelming creation of virtual relations. For Reason, however disinterested one may think it, has a utilitarian function. With the same activity, reason deduces a proposition from other propositions, and relations between natural phenomena. If quantitative relations, whish are the object of mathematics, agree with the laws of nature as well as with the laws of thought, it is because of the conformity of the order in nature and the order in thought. We are adapted to our environment, to the world in which we live, in such a way as to make possible not only onr material living, but also our 
intellectual development. Neither our sensible organs, nor our intellect could attain their normal development if there were an incompatibility between human mind and the external world, if our previsions, based on undeniable observations, led us to untrue conclusions with regard to similar observations. If science is power, it is because our reason, in its attempt to comprehend nature, gives us thereby the necessary means to act on it. We are therefore convinced that the universe has its laws and. that we are able to penetrate them: we are convinced that the external world cannot change its laws, in the same way as we cannot change the laws of thought; we feel, so to speak, that Reality binds us with it, in an insuperable circle.

In this stage of reflection, the thinker has to face a last aspect of the problem, which is by far the most alarming. If the alliance between mathematics and physics is undeniable, does science explain things as they are? Are science and reality identical? The great physicist, Duhem, denied that science can explain everything. For him, a physical theory is but an attempt towards a symbolic representation of Reality, and not an explanatory inquiry about the real. "A physical theory," he states, "is an abstract system which sums up and classifies logically a group of experimental laws, without pretending to explain them." An eminent mathematician and philosopher, Professor Le Roy, goes further, when he says that not only physical theories are symbolic and conventional, but that scientific facts themselves are mere creations of our mind. For him, science is made up of conventions, whence its apparent truth. But scientific facts, and a fortiori scientific laws, are artificial creations of the scientist himself. Science, therefore, cannot teach us anything about reality; it can only be used as a rule of action.

We could hardly expect any other from a distinguished follower of Bergson. Yet at the same time we maintain that it

* Dubem, La Thtorie Physique: Sa Structure. 
is impossible for science to be anti-intellectualistic: science must be intellectualistic, or it will not be at all. We take science as a logical classification of facts, as a means of putting together certain observations which are apparently separated, although they are really linked by some hidden but natural necessity. But a logical classification is an explanation by the natural causes; because its object is not to reveal to us any order in nature, save the order existing in things, the intimate relations between objects of reality; if possible the plan of nature itself. It is not the privilege of reason to create physical laws, but only to utilize them when discovered. We cannot pretend however, that science gives us the comprehension of the true essence of things. If we get every day nearer and nearer to the knowledge of reality, we must confess at the same time. that we are still, with respect to the ultimate mechanism of things, in the position of an engineer towards a machine of which a few organs only are visible, the remainder being still inaccessible to him. The reason of our ignorance is simple: it is the materiality of human means of perception, which hinders our comprehension of the ultimate nature of things. Besides, whatever has relations to things distinct from itself, could not be what it is, if those different things did not exist; and therefore, we cannot know a thing as it is, unless we know all its relations to all the other things in the universe. We cannot hope then to penetrate adequately the essence of things, although we must be convinced, with the great mineralogist Lapparent, that science progresses by successive approximations towards an adequate explanation of the relations between sensible things. Scientific certitude may be considered as asymptotic, because of the hypothetical character of scientific theories. Science and reality are not, therefore, congruent: the evolution of our knowledge and the nature of things are in the relation of a science-curve to its reality-asymptote.

But at the same time, we must acknowledge that every day 
we make a now conquest over the unknown, which remains for us a definite acquisition. Although scientific theories cannot be looked upon as an adequate knowledge of Nature, because Reality is necessarily refracted by the mental factors of our general constructions, yet our knowledge of particular facts and even of limited groups of facts related between themselves, can be materially true, independently of the scientific theories with which they may agree. If then, the evolution of the various physical theories from the early Greek philosophers up to the present day seems to prove a constant failure of theoretical science, we must realize that, amongst what are considered to be ruins in an old physical theory, there remains a certain invariant which makes the very value of the now theories. Light, for instance, is subject to gravitation; Newton knew it and gave the figures for the deflection of rays of light grazing the sun. Einstein, by means of a new theory, corrects Newton's formula, as we know. But whatever be the exact amount of that deflection, whatever be the primitive assumptions of the theories predicting that deflection, the fact is there; the deflection of the rays of light -grazing the sun is an invariant which subsists whatever be the fortune of the physical systems which try to account for it. So, by calling a fact "desoxydation" or "phlogistication" we do not change the fact as such: its expression only is altered. The same again, we may call a geometrical entity a "Euclidian circumference" or a "Riemannian straight line" in the antipodal system, without altering its very nature. In other words, the essence of a thing does not change when its meaning is expressed in English, in French or in German.

We do not go, however, as far as to say that science is a well-made language, as Locke contends; for us, science is not only a nominalistic system of coherent relations between indefinables, but the expression of true relations between real objects. Sensible things, for which the word "object" has been invented, are really objects and not fugitive and unseizgble appearances. When we ask then what is the value of science, 
we do not mean does science enable us to comprehend the very essence of things, but does it enable us to understand the true relations of things? And science has lived long enough to prove the stability of its particular constructions. As Poincare says, through the evolution of scientific systems, there is something which is always there, always present, definitely acquired, and that something is the essential.

It could not be otherwise. For changes do not happen in the laws of nature or in natural facts, but in the way in which we conceive and express them. Reality is immutable; our interpretations of it are, however, conditioned by the necessity and the adequacy of the data on which we base our solutions. One may argue here that, if the world is rational, we ought not to make mistakes in the expression of its laws. But if we know thoroughly the laws of our mind, we are not quite sure about the laws of nawure. The materiality of our being is an obstacle to our immediate knowledge of things as they are. We have to reason first about appearances, and, naturally, commit errors. Our convictions, even reasoned, are not a sufficient guarantee of truth.

We conclude, then, by saying, that science has a value independently of our reason. The reality of the external world is certainly independent of the eyes which see it, of the hands which touch it, of thought itself. Reality is not created in and by empirical experience; it exists ready-made outside and prior to experience. In that sense, we can say that the content of science is reality. So that if, per impossibile, mankind were annihilated, science would undoubtedly disappear as such. But its content would still be there; nature would continue its performance according to the same laws; the spectators only would be missing. 